INPLASY

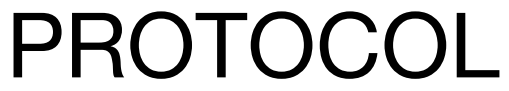

To cite: Ying et al. Perineural Invasion is an Indication of Adjuvant Chemotherapy in Node Negative Colorectal cancer. Inplasy protocol 2021120103. doi:

10.37766/inplasy2021.12.0103

Received: 23 December 2021

Published: 23 December 2021

Corresponding author:

Weiwen Hong

231723605@qq.com

Author Affiliation:

Taizhou first people's Hospital.

Support: Taizhou Science and Technology.

Review Stage at time of this submission: Piloting of the study selection process.

Conflicts of interest:

None declared.

\section{Perineural Invasion is an Indication of Adjuvant Chemotherapy in Node Negative Colorectal cancer}

Ying, $\mathrm{H}^{1}$; Shao, J2; Xu, X3; Yu, W4; Hong, W55.

Review question / Objective: Perineural invasion (PNI) is a possible route for metastatic spread in various cancer types, including colorectal cancer (CRC). PNI is linked to poor prognosis. For patients with lymph node positive colorectal cancer, a number of large-scale RCT studies have confirmed that they can benefit from chemotherapy, but there are still many controversies about whether colorectal patients with negative lymph nodes need adjuvant chemotherapy. At present, there is a general consensus that patients with stage II colorectal cancer who have risk factors such as PNI+ need chemotherapy. However, there are many recent literatures that show that patients with stage II colorectal cancer with nerve invasion risk factors can not prolong the OS and DFS of patients. At the same time, chemotherapy increases the toxicity, economic and mental burden of patients. Therefore, we hope to write this review to summarize the current research findings and provide some clinical guidance on whether patients with lymph node negative colon cancer who have perineural invasion should receive chemotherapy.

Condition being studied: Patients with high-risk such as PNI+ stage II colon cancer (CC) are recommended to undergo adjuvant chemotherapy (ACT). However, whether such patients can benefit from ACT remains unclear. And recently studies shown that, ACT had no significant benefit among patients with PNI.

INPLASY registration number: This protocol was registered with the International Platform of Registered Systematic Review and Meta-Analysis Protocols (INPLASY) on 23 December 2021 and was last updated on 23 December 2021 (registration number INPLASY2021120103).

\section{INTRODUCTION}

Review question / Objective: Perineural invasion (PNI) is a possible route for metastatic spread in various cancer types, including colorectal cancer (CRC). PNI is linked to poor prognosis. For patients with lymph node positive colorectal cancer, a number of large-scale RCT studies have confirmed that they can benefit from 
chemotherapy, but there are still many controversies about whether colorectal patients with negative lymph nodes need adjuvant chemotherapy. At present, there is a general consensus that patients with stage II colorectal cancer who have risk factors such as PNI+ need chemotherapy. However, there are many recent literatures that show that patients with stage II colorectal cancer with nerve invasion risk factors can not prolong the OS and DFS of patients. At the same time, chemotherapy increases the toxicity, economic and mental burden of patients. Therefore, we hope to write this review to summarize the current research findings and provide some clinical guidance on whether patients with lymph node negative colon cancer who have perineural invasion should receive chemotherapy.

Condition being studied: Patients with high-risk such as PNI+ stage II colon cancer (CC) are recommended to undergo adjuvant chemotherapy (ACT). However, whether such patients can benefit from ACT remains unclear. And recently studies shown that, ACT had no significant benefit among patients with PNI.

\section{METHODS}

Search strategy: PUBMED: ((Colorectal Neoplasms[MeSH Terms])OR(Colorectal Neoplasm[Title/Abstract])OR(Neoplasm, Colorectal[Title/Abstract])OR(Neoplasms, Colorectal[Title/Abstract])OR(Colorectal Tumors[Title/Abstract])OR(Colorectal Tumor[Title/Abstract]) OR (Tumor, Colorectal[Title/Abstract])OR(Tumors, Colorectal[Title/Abstract])OR(Colorectal Cancer[Title/Abstract]) OR(Cancer, Colorectal[Title/Abstract])OR(Cancers, Colorectal[Title/Abstract])OR(Colorectal Cancers[Title/Abstract])OR(Colorectal Carcinoma[Title/Abstract])OR(Carcinoma, Colorectal[Title/Abstract])OR(Carcinomas, Colorectal[Title/Abstract])OR(Colorectal Carcinomas[Title/Abstract])OR(Colonic Neoplasms[MeSH Terms])OR(Colonic Neoplasm[Title/Abstract])OR(Neoplasm, Colonic[Title/Abstract])OR(Neoplasms, Colonic [Title/Abstract]) OR (Colon
Neoplasms[Title/Abstract])OR(Colon Neoplasm[Title/Abstract])OR(Neoplasm, Colon[Title/Abstract])OR(Neoplasms, Colon[Title/Abstract])OR(Cancer of Colon [Title/Abstract]) OR (Colon Cancers [Title/Abstract]) OR(Colon Cancer[Title/Abstract])OR(Cancer, Colon[Title/Abstract]) OR(Cancers, Colon[Title/Abstract])OR(Cancer of the Colon[Title/Abstract]) OR(Colonic Cancer[Title/Abstract]) OR(Cancer, Colonic [Title/Abstract])OR(Cancers, Colonic[Title/Abstract])OR(Colonic Cancers[Title/Abstract]) OR(Colon A d e n o c a r c i n o m a [ T i t l e / Abstract])OR(Adenocarcinoma, Colon[Title/ Abstract]) OR (Adenocarcinomas, Colon[Title/Abstract]) OR(Colon A d e no c a r c i nom as [ T i t le/ Abstract])OR(Rectal Neoplasms[MeSH Terms])OR(Neoplasm, Rectal[Title/ Abstract])OR(Rectal Neoplasm[Title/ Abstract])OR(Rectum Neoplasms[Title/ Abstract])OR(Neoplasm, Rectum[Title/ Abstract])OR(Rectum Neoplasm[Title/ Abstract])OR(Rectal Tumors[Title/ Abstract])OR(Rectal Tumor[Title/ Abstract]) OR(Tumor, Rectal[Title/ Abstract])OR(Neoplasms, Rectal[Title/ Abstract])OR(Cancer of Rectum[Title/ Abstract])OR(Rectum Cancers[Title/ Abstract])OR(Rectal Cancer[Title/ Abstract])OR(Cancer, Rectal[Title/ Abstract])OR(Rectal Cancers[Title/ Abstract])OR(Rectum Cancer[Title/ Abstract])OR(Cancer, Rectum[Title/ Abstract])OR(Cancer of the Rectum[Title/ Abstract])) AND ((Chemotherapy, Adjuvant[MeSH Terms])OR(Drug Therapy, Adjuvant[Title/Abstract]) OR(Adjuvant Chemotherapy[Title/Abstract])OR(Adjuvant Drug Therapy[Title/Abstract])OR(Therapy, Adjuvant[Title/Abstract])OR(Adjuvant Therapy[Title/Abstract])) AND ((perineural invasion[Title/Abstract])).

Participant or population: Patients with $\mathrm{PNI}+$ lymph node positive colorectal cancer, including I, II stage with the risk factor of PNI.

Intervention: Adjuvant chemotherapy.

Comparator: Observation. 
Study designs to be included: RCT, retrospective cohort, prospective cohort.

Eligibility criteria: We included studies that met the following requirements:(1) patients who underwent radical resection of $\mathrm{CC}$, and stage II CC was confirmed by pathologic or histologic examination after surgery; (2) the article assessed the relationship between patients with highrisk factor of perineural invasion receiving ACT and overall survival (OS) or disease free survival (DFS); (3) full text in English; and (4) sufficient information to extract hazard ratios (HRs) and their 95\% confidence intervals (Cls). We excluded case reports, letters, conference summaries, duplicate publications, and papers from which we could not extract the relevant data.

Information sources: We systematically retrieved articles from PubMed, the Cochrane Library, web of science and Embase published in English up to December 15, 2021. We also searched the references of relevant articles identified in our studies.

Main outcome(s): The main outcome is $\mathbf{5}$ years overall survival(OS) or 5 years disease free survival(DFS).

Data management: Two researchers will separately extract the following informations by using data extraction forms which have been prepared in advance: (1) Research Characteristics: Year of publication, Journal, title, information of the author. (2) Participants' basic information: age, stage of cancer, country, sample size, tumour location, following time. (3) Study methods: prospective cohor, retrospective cohort, randomized controlled trial, clinical trial. (4) Intervention: adjuvant chemotherapy. (5) Outcomes measurement: outcome(OS, DFS) are measured in hazard radio(HR).

Quality assessment / Risk of bias analysis: Two researchers extracted relevant data. Quality was assessed by the NOS. Articles with an NOS score $>6$ points (on a scale of 0 to 9 ) were considered high quality. Using
Begg and Egger tests to assess the publication bias.

Strategy of data synthesis: StataMP $\mathbf{1 6 . 0}$ will be used to conduct the following data analysis. The risk ratio (RR) with 95\% confidence interval $(\mathrm{Cl})$ will be used when the measured outcomes are dichotomous data. If we use the same measurement instrument and the measured outcomes are continuous data, we will use the Weighted Mean Difference (WMD) with 95\% CI. If we use different measurement instruments, the Standardized Mean Difference (SMD) with 95 percent confidence interval will be used. To investigate heterogeneity, the $\times 2$ and 12 tests will be used. We will use the fixedeffect model when the heterogeneity is not obvious ( $P>0.1$ or 120.1 or $12<50 \%$ ), the random-effect model is chosen, and subgroup analysis or sensitivity analysis is performed to look for potential causes of intergroup heterogeneity. A descriptive analysis is necessary to be carried out if the heterogeneity is too significant.

Subgroup analysis: If the included trials have significant heterogeneity, a subgroup analysis is required to reduce heterogeneity between groups due to differences in massage methods, cancer stage, sample size, tumour location and so on.

Sensitivity analysis: If necessary, a sensitivity analysis will be performed to determine whether the meta-analysis' conclusions are stable or reliable by excluding low-quality studies.

Language: English.

Country(ies) involved: China.

Keywords: Perineural invasion, Colorectal cancer, Node negative, Adjuvant chemotherapy, Protocol, Systematic review.

Contributions of each author:

Author 1 - Hongan Ying - in charge of the study's conception and design; 2. perform 
the study selection, data extraction and statistical analyses.

Email: 269073862@qq.com

Author 2 - Jinfan Shao - The author perform the study selection, data extraction and statistical analyses. 2. The author read, provided feedback and approved the final manuscript.

Email: sjfimba@sina.com

Author 3 - Xijuan Xu - The author is responsible for the draft of the manuscript. Email: 465590555@qq.com

Author 4 - Wenfeng Yu - The author is responsible for the draft of the manuscript. Email: 1197595560@qq.com

Author 5 - Weiwen Hong - The author is responsible for the conception and design the study and contributed to a critical revision of the manuscript.

Email: 231723605@qq.com 